\title{
Diagnosis of urinary tract infections: need for a reflective rather than reflexive approach
}

\author{
Mohamad G. Fakih MD, MPH ${ }^{1,2}$ (1), Sonali D. Advani MBBS, MPH ${ }^{3}$ (1) and Valerie M. Vaughn MD, MSc ${ }^{4,5}$ \\ ${ }^{1}$ Care Excellence, Ascension Healthcare, St Louis, Missouri, ${ }^{2}$ Wayne State University School of Medicine, Detroit, Michigan, ${ }^{3}$ Section of Infectious Diseases, Yale \\ School of Medicine, New Haven, Connecticut, ${ }^{4}$ Department of Internal Medicine, University of Michigan Medical School, Ann Arbor, Michigan and ${ }^{5} \mathrm{Center}$ for \\ Clinical Management Research, VA Ann Arbor Healthcare System, Ann Arbor, Michigan
}

To the Editor-We read with great interest the study by Munigala et $\mathrm{al}^{1}$ on the impact of changing urine testing orderables on the number and positivity of urine cultures. With the increased attention to catheter-associated urinary tract infection (CAUTI), the vast majority of hospitals in the United States have steered their attention to "stewardship of culturing" to reduce the detection of asymptomatic catheter-associated bacteriuria. In this retrospective study, the authors evaluate changing urine culture reflex testing in order sets and engaging clinicians regarding the change. Prior to the intervention, a urinalysis would be reflexed to culture if it included any protein, blood, nitrite, or leukocyte-esterase positive results. With the intervention, the urinalysis reflexed to culture only if it was positive for either nitrite or leukocyte esterase. A $45 \%$ reduction in urine cultures performed was observed after implementation, although catheter-associated bacteriuria and CAUTI rates did not significantly change.

The main intervention was to exclude urinalysis parameters of proteinuria or blood, both elements not typically associated with bacteriuria, from reflexing to cultures. This raises the issue that there is no national standard for "reflex urine culture" criteria in hospitalized patients. ${ }^{2}$ A previous study that incorporated reflex urine cultures relied on arbitrary triggers (white cells $>10$ per high-power field) of the urinalysis to reflex to culture, leading to a reduction in catheter-associated bacteriuria but not antibiotic use. ${ }^{3}$ Other successful efforts to reduce culturing and CAUTI have focused on engaging clinicians with protocols on how to address fever without requiring strict implementation of urinalysis reflex to cultures. ${ }^{4}$ Whether any of the interventions have improved the diagnosis of urinary tract infection (UTI) remains unknown.

Notably, the reduction in urine cultures from Munigala's study were disproportionately related to catheterized patients compared to clean-catch samples ( $76 \%$ vs $38 \%$ decrease). This finding indicates a potential clinician bias toward ordering fewer cultures in catheterized patients during the intervention phase in a population that is more likely to develop bacteriuria and pyuria because of instrumentation and, thus, more likely to have abnormal urinalyses.

Bacteriuria was common, with $>25 \%$ of urine cultures being positive, identified as either $>100,000$ colony-forming units per milliliter $(\mathrm{CFU} / \mathrm{mL})$ for clean-catch collection or $>10,000 \mathrm{CFU} /$ $\mathrm{mL}$ for catheterized patients. The extent of bacteriuria likely would have been even higher if cultures with lower colony counts were

Author for correspondence: Mohamad G. Fakih, Email: Mohamad.Fakih@ascension.org Cite this article: Fakih MG, et al. (2019). Diagnosis of urinary tract infections: need for a reflective rather than reflexive approach. Infection Control \& Hospital Epidemiology, 40: 834-835, https://doi.org/10.1017/ice.2019.98 included. The number of positive cultures per 1,000 patient days decreased by more than one-third, but it is not clear whether the improvements were related to a general reduction in urine culturing compared to better identification of symptomatic patients. Although a negative urinalysis may have a high negative predictive value for bacteriuria and infection, an "abnormal" result does not indicate infection. ${ }^{5}$ An assessment of the pretest probability (ie, symptoms and signs) is key to better identify urinary tract infection. Reflex urine cultures used in isolation enhance the identification of bacteriuria (both symptomatic and asymptomatic) but not necessarily the diagnosis of UTIs. Although its use has been well validated in outpatient setting, it may not be an optimal strategy for the inpatient or catheterized populations.

Before we declare whether reflex urine cultures "save the day," we need to address the importance of reviewing signs and symptoms suggestive of a UTI prior to urine testing. UTI is suspected based on focal signs and symptoms attributed to the urinary tract, or systemic findings in patients at risk with no other apparent source of infection. Asymptomatic bacteriuria is common, especially in the elderly and in catheterized patients. ${ }^{6}$ Hence, we endorse a stepwise approach, as recommended by Morgan et al, ${ }^{7}$ targeting ordering, collection, processing, and reporting of urine cultures (Fig. 1). In the ordering (preanalytic) stage, testing should be restricted to patients with symptoms referable to the urinary tract (with some exclusions as per guidelines) and inappropriate practices, such as indiscriminate 'pan-culturing,' should be discouraged. ${ }^{8-10}$ In patients with nonspecific symptoms like delirium or fever, it is important to first rule out an alternate cause of a patient's symptoms before considering urine cultures. The collection (preanalytic) stage focuses on urine collection techniques to reduce contamination. The processing (analytic) stage relies on optimizing reflex testing by only targeting this test to patients with UTI symptoms and by identifying optimal parameters for reflexing. The reporting (postanalytic) stage includes framing diagnostic results to help providers accurately interpret and respond to results.

As providers, we should be cognizant that the value of both urinalysis and urine culture depends on the clinical condition of the patient. For those who order, collect, and interpret urinalyses, this means moving away from a reflexive approach to testing and treatment, toward a reflective one. Although the "knee jerk" response to evaluating a patient with a fever or altered mental status may be obtaining a urinalysis, there are myriad other causes of these symptoms in hospitalized patients which are potentially missed by focusing on the urine. Similarly, reflexive treatment of "positive" urine cultures based on "positive" urinalyses leads to overtreatment, especially in groups with high pretest probability for 


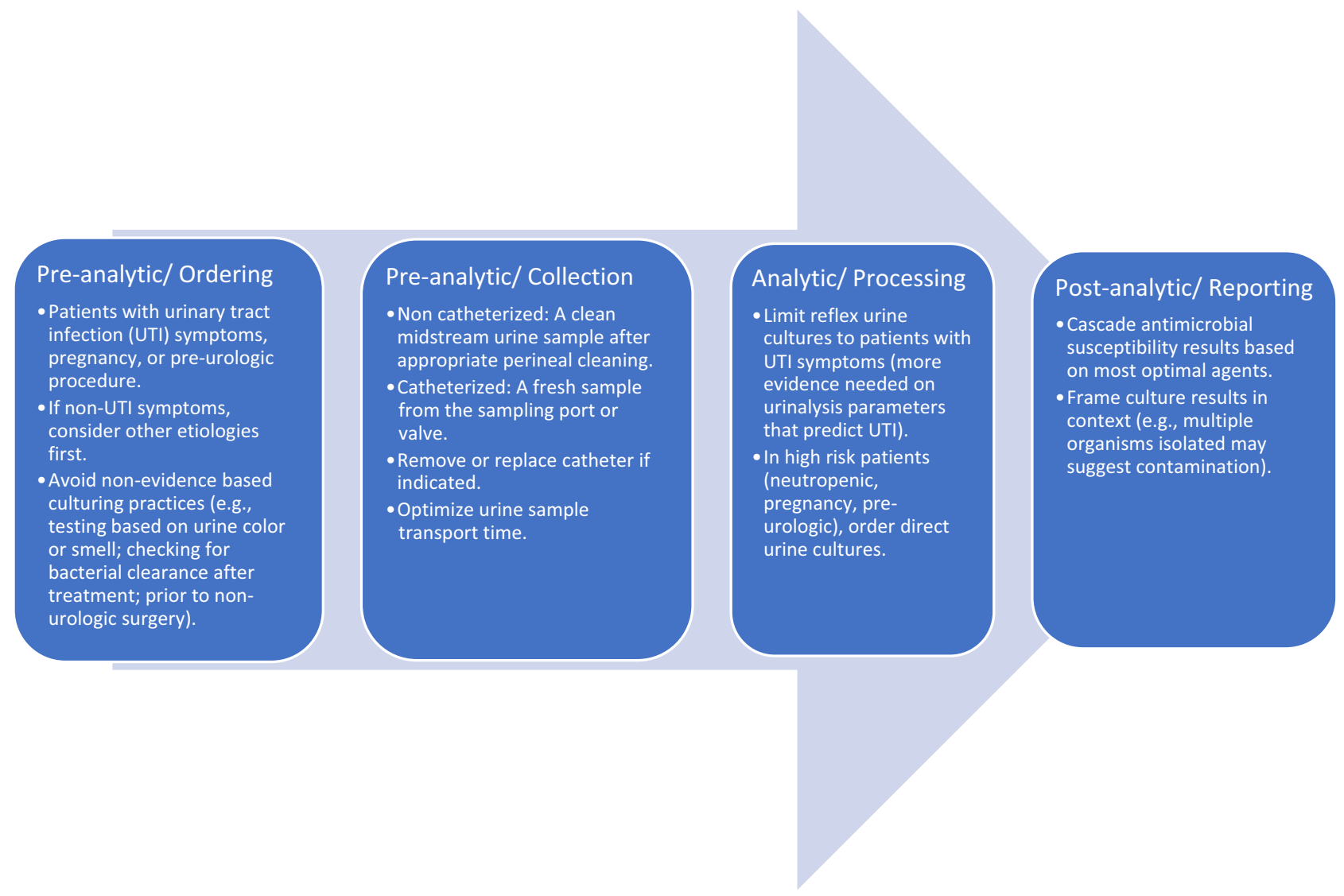

Fig. 1. Stages of diagnostic stewardship interventions with urine cultures.

bacteriuria. And as long as we are asking providers to be more reflective than reflexive-perhaps it is time to do the same of urinalyses, too.

Author ORCIDs. Mohamad G Fakih, (D) 0000-0002-2258-8015; Sonali D Advani, (D) 0000-0001-5162-6482

\section{Acknowledgments. None}

Financial support. No financial support was provided relevant to this article.

Conflicts of interest. All authors report no conflicts of interest relevant to this article.

\section{References}

1. Munigala S, Rojek R, Wood $\mathrm{H}$, et al. Effect of changing urine testing orderables and clinician order sets on inpatient urine culture testing: analysis from a large academic medical center. Infect Control Hosp Epidemiol 2019;40:281-286.

2. Sullivan KV, Morgan DJ, Leekha S. Use of diagnostic stewardship practices to improve urine culturing among SHEA research network hospitals. Infect Control Hosp Epidemiol 2019;40:228-231.

3. Sarg M, Waldrop GE, Beier MA, et al. Impact of changes in urine culture ordering practice on antimicrobial utilization in intensive care units at an academic medical center. Infect Control Hospital Epidemiol 2016;37: 448-454.

4. Mullin KM, Kovacs CS, Fatica C, et al. A multifaceted approach to reduction of catheter-associated urinary tract infections in the intensive care unit with an emphasis on "stewardship of culturing." Infect Control Hosp Epidemiol 2017;38:186-188.

5. Humphries RM, Dien Bard J. Point-counterpoint: reflex cultures reduce laboratory workload and improve antimicrobial stewardship in patients suspected of having urinary tract infections. J Clin Microbiol 2016;54:254-258.

6. Nicolle LE, Bradley S, Colgan R, Rice JC, Schaeffer A, Hooton TM. Infectious Diseases Society of America guidelines for the diagnosis and treatment of asymptomatic bacteriuria in adults. Clin Infect Dis 2005;40:643-654.

7. Morgan DJ, Malani P, Diekema DJ. Diagnostic stewardship-leveraging the laboratory to improve antimicrobial use. JAMA 2017;318:607-608.

8. Fakih MG, Khatib R. Improving the culture of culturing: critical asset to antimicrobial stewardship. Infect Control Hosp Epidemiol 2017; 38:377-379.

9. Hooton TM, Bradley SF, Cardenas DD, et al. Diagnosis, prevention, and treatment of catheter-associated urinary tract infection in adults: 2009 International clinical practice guidelines from the infectious diseases society of America. Clin Infect Dis 2010;50:625-663.

10. Vaughn VM, Chopra V. Revisiting the panculture. BMJ Qual Saf 2017;26:236-239. 